\title{
Towards athermal optically-interconnected computing system using slotted silicon microring resonators and RF-photonic comb generation
}

\author{
Linjie Zhou • Ken Kashiwagi $\cdot$ Katsunari Okamoto • \\ R.P. Scott • N.K. Fontaine · Dan Ding • \\ Venkatesh Akella $\cdot$ S.J.B. Yoo
}

Received: 3 October 2008 / Accepted: 16 December 2008 / Published online: 14 February 2009

(C) The Author(s) 2009. This article is published with open access at Springerlink.com

\begin{abstract}
We report that completely athermal design of a slotted silicon waveguide is possible by combining the negative thermo-optic (TO) coefficient of, for example, polymethyl methacrylate (PMMA) with the positive TO coefficient of silicon. When used in a microring resonator structure, the filled overcladding slotted waveguide and the unfilled (air-filled) overcladding slotted waveguide can both achieve athermal characteristics. Simulations indicate a wide range of realizations with proper design parameters of the slotted waveguides, namely, the silicon strip and slot widths. Preliminary experimental results on fabricated devices demonstrate that the temperature dependence is reduced from $91 \mathrm{pm} /{ }^{\circ} \mathrm{C}$ for a regular microring resonator to $52 \mathrm{pm} /{ }^{\circ} \mathrm{C}$ for the PMMA-clad microring resonator. Completely athermal realization is expectable in similar devices with improved fabrication techniques. For the external optical source, we demonstrate a stable $3.5 \mathrm{THz}$ wide $(175$ modes $\times 20 \mathrm{GHz})$ optical comb source with nearly flat spectral phase. Adjustable mode spacing and wavelength tunability across the C-band are maintained so that comb lines can be matched to the specified wavelength grid of the computing system. With such schemes, temperature controls of individual optical components in the optically intercon-
\end{abstract}

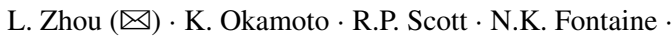

D. Ding · V. Akella $\cdot$ S.J.B. Yoo

Department of Electrical and Computer Engineering, University

of California, Davis, CA 95616, USA

e-mail: 1jzhou@ucdavis.edu

Fax: +1-530-7528428

K. Kashiwagi

Department of Electronic Engineering, Graduate School of Engineering, University of Tokyo, Bunkyo, Tokyo 113-0033, Japan nected computing chips become unnecessary, greatly reducing the complexity of the computing system.

PACS 42.82.Ds $\cdot$ 42.79.Ta $\cdot$ 42.72.Ai

\section{Introduction}

In 1967, Amdahl proposed a set of rules of thumb for computer architects that have withstood the test of time. One such rule of thumb is that a balanced computing system [1] should be capable of providing one byte of memory and one byte per second of memory bandwidth for each instruction per second of computation. Building balanced computing systems in the terascale era is challenging because of pin limitations, and poor scalability of bandwidth and memory capacity with off-chip electrical interconnects between the CPU and memory subsystem. Optics provides numerous benefits over electrical (copper) interconnects [2, 3]. Its high carrier frequency of $10^{15} \mathrm{~Hz}$ results in no signal degradation with modulation frequency and it has low propagation loss (less than $0.1 \mathrm{~dB} / \mathrm{km}$ for glass fiber). Photonic interconnects provide ultra-high throughput, minimal access latencies, and low power dissipation that remains independent of capacity and distance. Optical communication links based on nanoscale silicon photonics have shown the ability to operate at $100 \mathrm{fJ} / \mathrm{bit}$, with future modifications for bringing it down to $10 \mathrm{fJ} / \mathrm{bit}[4,5]$. In addition to the energy efficiency, many of the fundamental physical problems of electrical interconnects are directly addressed within the optical technology platform, including precise clock distribution, bit rate transparency, and power reduction, without concerns for impedance, crosstalk, voltage isolation, pin inductance, signal distortion, and repeater-induced latency $[2,6]$. 
Fig. 1 An example of future multi-core computing system-on-a-chip with multi-core processor array (bottom layer), memory (middle layer), and optical layer (top layer) including optical resonators, modulators, and plasmonic antennas

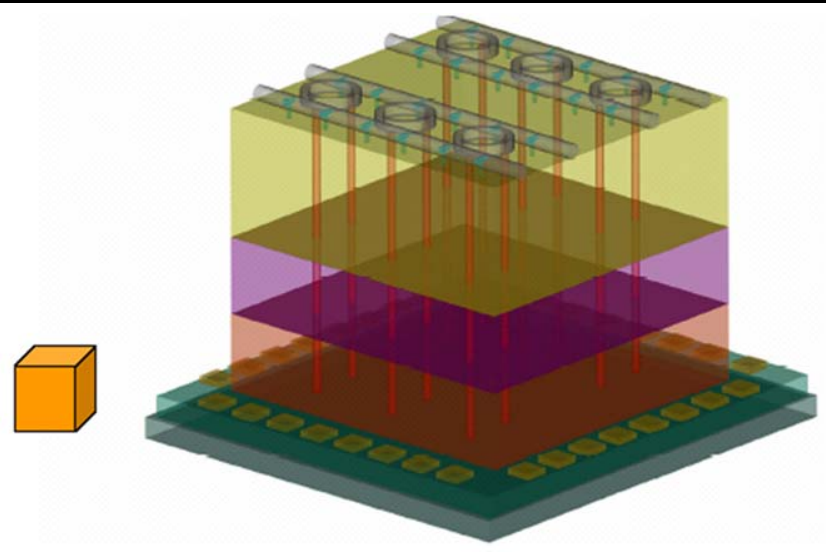

The opportunity for optical interconnects $[7,8]$ in board-toboard and rack-to-rack communications is already well documented. Recent work from UC Davis $[9,10]$ has shown that WDM based optical interconnects can be used in the CPU/DRAM interface to provide significantly higher bandwidth and increase memory capacity to realize balanced computing systems. Research at HP [11] and MIT [12] has shown that WDM based nanophotonic crossbar can be used in future high performance low power multicore processors and researchers at Cornell [13] showed that bus-based multiprocessors can take advantage of on-chip WDM based optical interconnects.

While wavelength division multiplexing (WDM) in CMOS compatible silicon photonics is clearly an advantageous method for realizing high-capacity optical interconnects, temperature sensitivities of the WDM components have been the main hurdle for deploying low-cost, compact, reliable, and low-power modules and subsystems. In particular, next generation computer communications will require micrometer and nanometer scale of such optical components to reside on computing chips $[14,15]$.

As an example, Fig. 1 illustrates a future terascale computing system with multi-core processor array (bottom layer), memory (middle layer), and optical layer (top layer) including optical resonators, modulators, and detectors. The optical frequency source is external to the chip, and provides multi-wavelength optical channels to the chip. The vertical vias consist of optical nanoantennas $[16,17]$ vertically funneling the optical signal down to a nanoscale detector. High index contrast silicon waveguides are very attractive for compatibility with the electronics [18, 19], small footprints, and strong modulation with current injection. However, silicon has a high thermo-optic coefficient which is one order larger than that of silicon-dioxide commonly used for photonic industry. Typical high performance multicore chips may have $\pm 10^{\circ} \mathrm{C}$ temperature variations, which would be far too large for predictable operation of the silicon-based WDM devices with typical temperature dependence of approximately $91 \mathrm{pm} /{ }^{\circ} \mathrm{C}$. Due to the sharp resonances of micro resonators, the ambient temperature variation should be controlled within $1^{\circ} \mathrm{C}$. In the past, attempts to achieve WDM device operations included placing micro thermal heaters, sensors, and controllers for each resonator [20] and placing strained layers [21].

Recently, slotted waveguides have attracted a lot of research interest, as they can confine light in the thin slot region and various materials can be filled as a convenient way to tailor their optical characteristics [22-38]. Filling the slot region with a material with the opposite thermal-optic coefficient to the core material will allows us to realize the temperature insensitive slotted waveguides, so that the optical waveguide characteristics will be maintained under temperature variations. Therefore, the temperature controls of the devices become unnecessary, greatly reducing the complexity and the operating power of the system. In this paper, we report a slotted silicon microring resonator with its slot filled with polymethyl methacrylate (PMMA) to reduce its resonance temperature dependence.

\section{Slotted silicon waveguide device structure}

Figure 2 shows the schematic structure of the slotted microring resonator on silicon-on-insulator (SOI) substrate. The inset shows the cross-sectional schematic of the slotted waveguide. The slotted waveguide has a slot region which is embedded within two silicon strips (high index regions). The device is upper-clad with lower refractive index material PMMA, which has the thermo-optic coefficient of the opposite sign compared to that of the silicon.

\section{Simulations of slotted silicon waveguides}

Assuming the refractive indices of $\mathrm{Si}, \mathrm{SiO}_{2}$ and PMMA are $3.48,1.46$ and 1.481 , and their thermal-optic (TO) coefficients are $1.84 \times 10^{-4}, 1.0 \times 10^{-5}$ and $-1.0 \times 10^{-4}$, respectively, we calculate effective refractive index $\left(n_{\text {eff }}\right)$ as 
Fig. 2 Schematic structure of a slotted microring resonator. Inset shows the cross-sectional schematic of the slotted waveguide
Fig. 3 (a)-(c) Simulated electric-field TE mode profiles for slotted waveguides with various slot widths.

(d) Temperature dependence of effective indices with various slot widths. (e) Simulated effective index temperature change slope as a function of slot width [39]
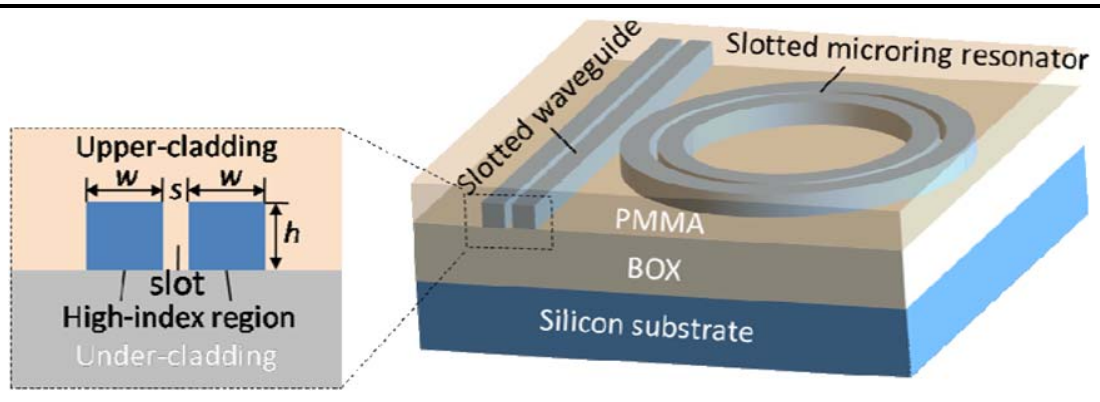

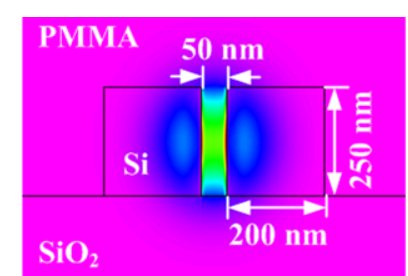

$$
\text { a }
$$

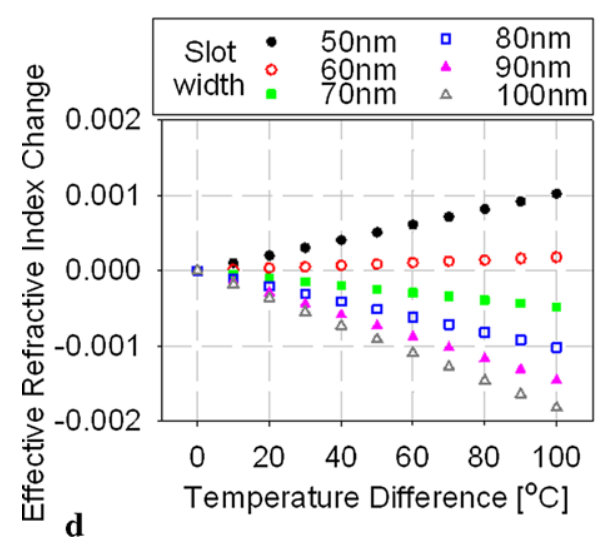

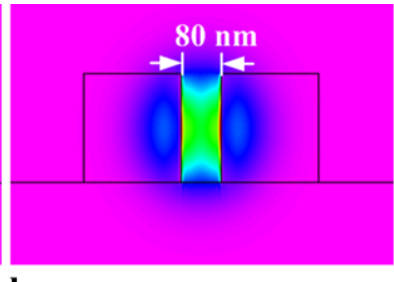

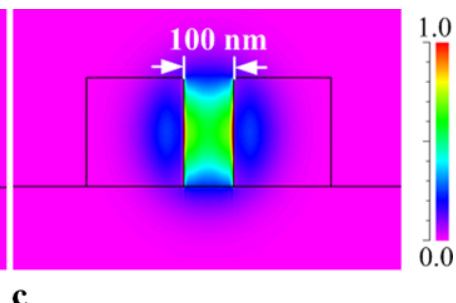

c

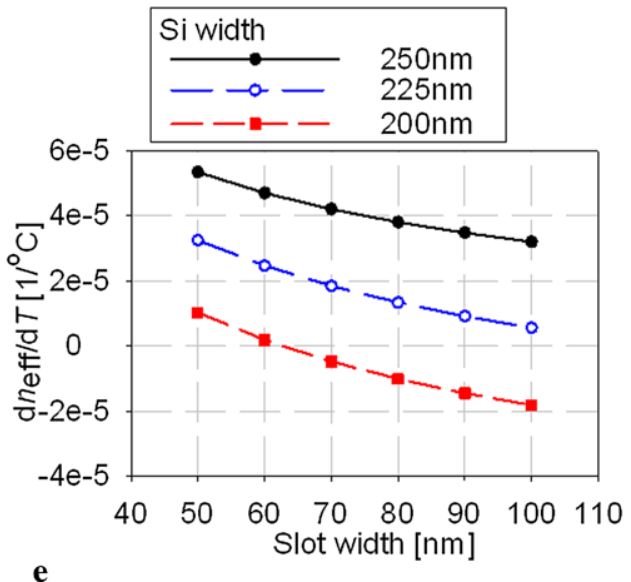

functions of silicon strip width $w$ and slot width $s$ using beam propagation method (BPM). Waveguide height is fixed at $250 \mathrm{~nm}$. Only TE mode is considered in this paper since light confinement in the slot region appears only in the TE mode.

Figures 3(a)-(c) show the simulated electric-field mode profiles for three slotted waveguides with slot widths of 50, 80 and $100 \mathrm{~nm}$, respectively. Figure 3(d) shows the temperature dependence of effective refractive index of the waveguide with several slot widths. In the case that the waveguide is temperature independent, effective refractive index change stays zero at any temperature. Figure 3(e) shows the slot width dependence of effective index change slope with three different silicon strip widths: 200, 225 and $250 \mathrm{~nm}$. The zero-crossing point is where the temperature insensitive waveguide structure is realized. For example, $\sim 62 \mathrm{~nm}$ slot width is the optimum for silicon strip width of $200 \mathrm{~nm}$. Under this athermal condition, increases in the silicon strip also leads to the increased slot width, relaxing the tolerance to the fabrication process.
In practice, it is difficult to fill the thin slot region with PMMA. However, temperature insensitive slotted waveguides can still be realized without filling any materials in the slot, or filling with air or vacuum [39]. Figure 4(a) show the schematic structure of PMMA-clad slotted waveguide with slot region filled with air. The refractive index of air is much smaller than that of PMMA and the light is much highly confined inside the slot region. Since the thermooptic coefficient of air is zero, the compensation can be realized only by PMMA in the upper-cladding region. Figure 4(b) shows the slot width dependence of effective index slope for the waveguide structure. For the case of silicon width of 200 and $220 \mathrm{~nm}$, temperature independence can be achieved with the slot width of 65 and $125 \mathrm{~nm}$, respectively.

\section{Fabrication of slotted silicon waveguides}

The device was fabricated using a silicon-on-insulator (SOI) wafer with top silicon layer thickness of $0.26 \mu \mathrm{m}$ and buried oxide (BOX) layer thickness of $2 \mu \mathrm{m}$. Figure 5 shows the 
Fig. 4 (a) Schematic of

PMMA-clad slotted waveguide with void left in slot (filled with air), (b) simulated effective index temperature change slope as a function of slot width
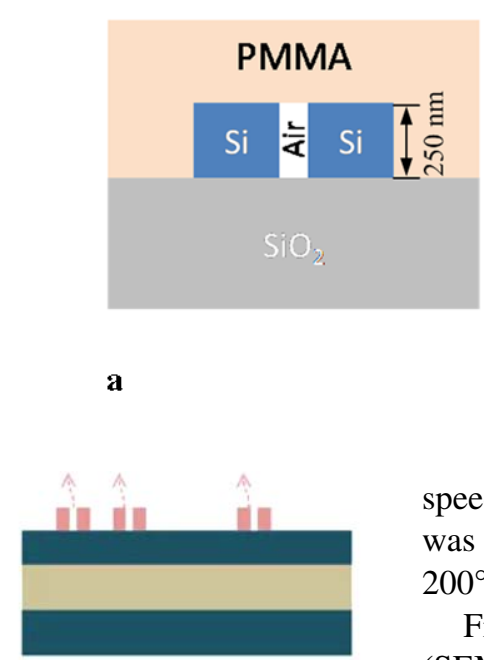

(4) UV-bake

(1) Begin with a SOI wafer

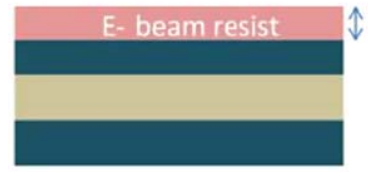

(2) E-beam resist coating

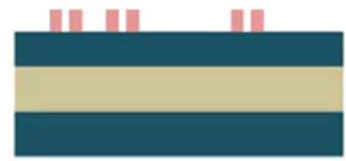

(3) E-beam lithography
$0.35 \mu \mathrm{m}$

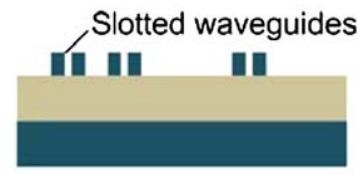

(5) RIE Dry etch

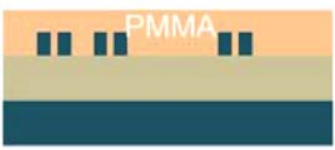

(6)PMMA coating
Fig. 5 Fabrication processes for the slotted microring resonator

schematics of the fabrication process flow. The wafer was first cleaned using Piranha solution $\left(\mathrm{H}_{2} \mathrm{SO}_{4}: \mathrm{H}_{2} \mathrm{O}_{2}=10: 1\right)$ to remove the organic residuals on the surface, and baked in Hexamethyldisilazane (HMDS) vapour oven at $150^{\circ} \mathrm{C}$ for 30 minutes to enhance the adhesion of the e-beam resist to the SOI substrate. The wafer was then coated with negative e-beam resist Microchem Corp. Ma-N 2403 at $2500 \mathrm{rpm}$ spinning speed with a thickness of $\sim 0.35 \mu \mathrm{m}$, and softbaked on a hotplate at $90^{\circ} \mathrm{C}$ for 1 minute. The patterns were exposed using Raith 150 e-beam lithography system with exposure dose of $80 \mu \mathrm{C} / \mathrm{cm}^{2}$ at $20 \mathrm{kV}$ acceleration voltage. The exposed wafers were developed in Microchem Corp. Ma-N 532 developer for 1 minute, and rinsed with deionized (DI) water to stop its development and blow-dried using $\mathrm{N}_{2}$ gas. UV-baking of the patterned photoresist enhanced its etch selectivity in favour of silicon etching during the subsequent $\mathrm{HBr}-\mathrm{Cl}_{2}$ gas-based reactive-ion-etching (RIE) in a Transformer Coupled Plasma (TCP) Lam Etcher. The RIE etching time was 40 seconds and the silicon etched depth is $\sim 0.25 \mu \mathrm{m}$.

To investigate the resonance temperature shift under PMMA cladding, we also spin coated the slotted microring resonator with 495k PMMA in anisole. The spinning

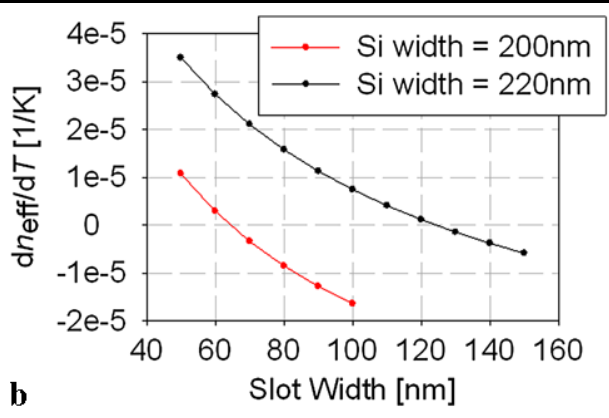

speed was $800 \mathrm{rpm}$ and resulting PMMA layer thickness was $\sim 0.5 \mu \mathrm{m}$. The device was finally baked on a hotplate at $200^{\circ} \mathrm{C}$ for 2 minutes.

Figure 6(a) shows the scanning electron microscope (SEM) image of the fabricated slotted microring resonator. The microring resonator diameter is $10 \mu \mathrm{m}$. The slotted waveguide width is $0.5 \mu \mathrm{m}$ with a $0.1-\mu \mathrm{m}$-wide slot in the center. The gap between the straight waveguide and the microring resonator is $0.2 \mu \mathrm{m}$. Figures $6(\mathrm{~b})$ and 6(c) show the zoom-in views of the slotted waveguide cross-section and the coupling region.

\section{Measurements}

We employed an external-cavity wavelength-tunable diode laser (1524-1576 nm, 300 kHz linewidth) to measure the device transmission spectra. The input laser beam was preamplified by an erbium doped fiber amplifier (EDFA) to $\sim 16 \mathrm{dBm}$ optical power and passed through a polarization controller to set the polarization to TE polarization (electric field parallel to the chip). The input light was butt-coupled to the chip through a tapered single-mode silica fiber. The chip was mounted on a thermoelectric cooling stage to control its temperature. At the output, an objective lens with numerical aperture $\mathrm{NA}=0.85$ was used to collect the output light. The collected light was finally detected by an InGaAs photodiode detector.

Figure 7(a) shows the measured TE transmission spectrum of the slotted microring resonator without PMMA cladding (air cladding). The measured $Q$ is $\sim 10^{3}$ and the resonance extinction ratio is $<3 \mathrm{~dB}$, most probably due to the increased losses in the slotted waveguide. Figures 7(b) and 7(c) show the resonance redshift of the slotted microring resonator with air cladding and PMMA cladding when temperature increases. In comparison, we also fabricated a regular wire waveguide-based microring resonator (add-drop filter configuration, air cladding) as the control device and measured its transmission spectrum as shown in Fig. 7(d). The wire waveguide in the control device has the identical width and height as the slotted waveguide. The control microring resonator has a diameter of $20 \mu \mathrm{m}$. Figure 7(e) shows its resonance temperature response. 
Fig. 6 (a) Scanning electron microscope (SEM) image of a slotted microring resonator, (b) zoom-in SEM image of the slotted waveguide cross-section, (c) zoom-in SEM image of the coupling region
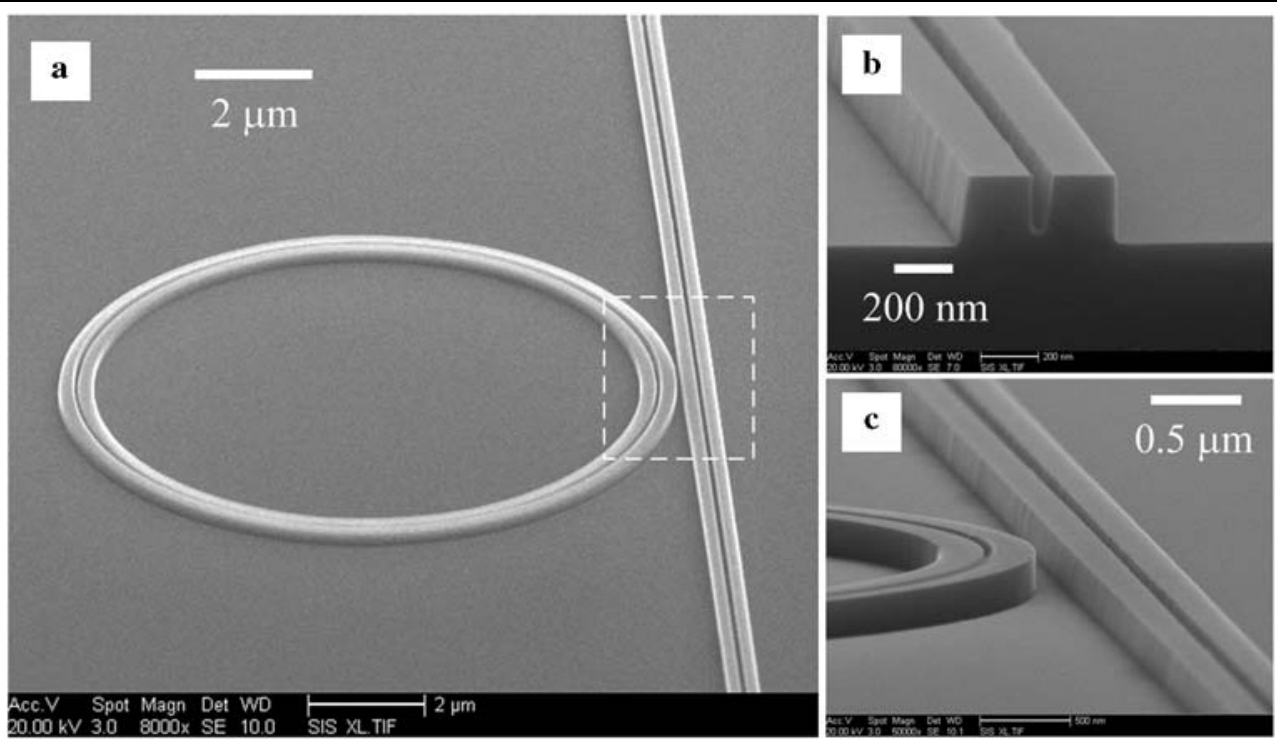
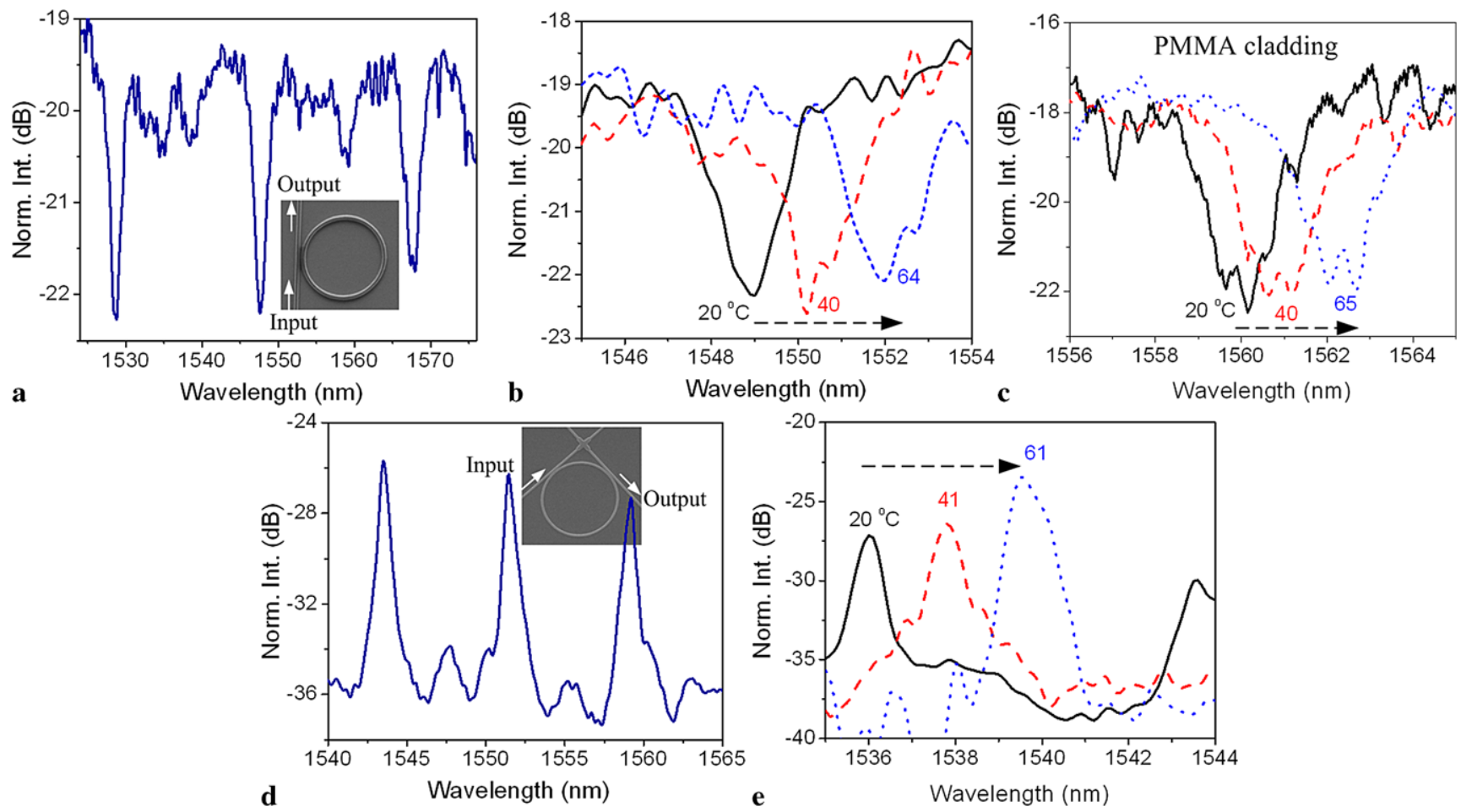

Fig. 7 (a) Measured transmission spectrum of the slotted microring resonator at room temperature, (b) resonance shifting of the slotted microring resonator at various temperatures, $(\mathbf{c})$ resonance shifting of the slotted microring resonator clad with PMMA at various temperatures,

Thus, for the above three cases, we can extract the resonance redshift value as a function of the temperature, as shown in Fig. 8. Linear curve fitting on the measured data indicates the resonance temperature shift rates of $91 \mathrm{pm} /{ }^{\circ} \mathrm{C}$ for the regular microring resonator, $67 \mathrm{pm} /{ }^{\circ} \mathrm{C}$ for the air-filled slotted microring resonator and $52 \mathrm{pm} /{ }^{\circ} \mathrm{C}$ for the PMAAclad slotted microring resonator. It shows that the slotted mi- (d) measured transmission spectrum of a regular microring resonator at room temperature, (e) resonance shifting of the regular microring resonator at various temperatures. Insets in (a) and (d) show the corresponding device top-view SEM images

croring resonator has significantly reduced temperature sensitivity compared to the regular microring resonator, which is expected since most of the optical intensity in the slotted waveguide is located in the air slot. Using PMMA cladding can compensate for the positive temperature coefficient of the silicon material and thus it can further reduce the resonance temperature sensitivity of the slotted microring res- 


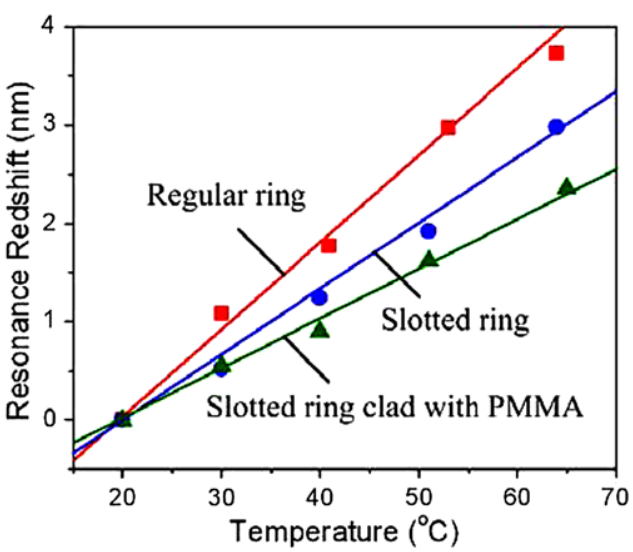

Fig. 8 Measured resonance redshift with temperature increase for three device: regular microring resonator, slotted microring resonator and slotted microring resonator clad with PMMA. The resonance redshift is normalized to $20^{\circ} \mathrm{C}$ case. Straight lines are the linear fitting

onator. We note that temperature dependence is not completely nulled even with PMMA cladding, probably due to the deviation of the slot dimension from the designed value (too thin) or due to PMMA not infiltrating to the slot region.

\section{External optical comb source}

Typical optical interconnects use low efficiency (below $30 \%$ ) lasers (e.g., VCSELs) in the blades or boards, where most of the power turns into heat in the system. Recently developed hybrid silicon modelocked lasers [40] indicate possible on-chip realization of optical comb sources, however, they suffer from strong instability in the comb wavelengths due to temperature variations and optical feedback, in addition to the aforementioned heat generation problems on chip. This paper will utilize an external laser source modulated in amplitude and phase using a dual-arm MachZehnder modulator to generate extremely broad and well controlled optical combs. Such optical comb sources can be considered as external optical power supplies to the computing system as depicted in Fig. 1.

The optical frequency comb generation (OFCG) described here maintains much of the flexibility and stability of the original OFCG [41] with 19 frequency comb generation across $190 \mathrm{GHz}$, yet provides 175 frequency comb generation across a $3.5 \mathrm{THz}$ wide optical spectrum with nearly flat spectral phase. It uses soliton-like compression in dispersion decreasing fiber (DDF) to generate the 175 optical modes. Figure 9 shows the arrangement used to generate a $3.5 \mathrm{THz}$ optical comb at $1550 \mathrm{~nm}$, which consists of an OFCG, compression fiber, optical amplifier and DDF. The comb generator utilizes a dual-electrode Mach-Zehnder modulator (DEMZM) to simultaneously amplitude and phase modulate (AM/PM) a single frequency laser (Anritsu MG9638A) and create a flattened comb with $20 \mathrm{GHz}$ spacing [42]. RF1 of the DEMZM is driven at $+27 \mathrm{dBm}$ and RF2 is driven at $+25 \mathrm{dBm}$. The output pulses of the OFCG are highly frequency chirped and the sign of the chirp is determined by the phase difference between RF1 and RF2. The sign of the output chirp is chosen so that standard single mode fiber (SMF28) will remove most of the chirp, thereby compressing the pulses to approximately $5 \mathrm{ps}$. An erbium-doped fiber amplifier (EDFA) amplifies the pulses to an average power of $+22 \mathrm{dBm}$ before they are launched into the DDF. The $1 \mathrm{~km}$ DDF (PriTel, DDF-400) has an input dispersion of $9.8 \mathrm{ps} / \mathrm{nm}$ and it linearly decreases to $2.1 \mathrm{ps} / \mathrm{nm}$ at the output [43]. It is optimized for transform-limited input pulses with widths of 2.5 to 5 ps. The optical comb was characterized with both a high resolution spectrometer (Agilent 83453A) and frequency resolved optical gating (FROG) [44]. The spectrum analyzer was set to a resolution of $250 \mathrm{MHz} /$ point and had a dynamic range of $\sim 45 \mathrm{~dB}$. FROG measurements of the comb were made using cross-correlation FROG (XFROG) with the optical modes spectrally resolved [44].

Figure 10 shows the pulse containing 175 optical modes after soliton-like compression in the DDF. These data were taken with a heterodyne-type, high-resolution spectrometer (250 MHz/point) that gives us the ability to easily resolve the spectral modes and verify that the optical signal-to-noise ratio (OSNR) had not significantly degraded.

Figure 11 shows the wavelength and repetition rate. The center wavelength of the laser was adjusted to $1530 \mathrm{~nm}$ for the data shown in Fig. 11(a). The higher output power at this wavelength allows significantly more power per mode to be generated than at $1550 \mathrm{~nm}$, with nearly 150 modes above $-20 \mathrm{~dB}$. The spectrum shown in Fig. 11(b) was taken with repetition rate set to $10 \mathrm{GHz}$. The reduced contrast is due to the resolution $(0.05 \mathrm{~nm}$ or $6.25 \mathrm{GHz})$ of the optical spectrum analyzer (OSA). However, over 300 modes are shown in Fig. 11(b). One characteristic of DDF compression is apparent in all of the DDF output spectra: dips in the spectrum near the center wavelength. These dips appear due to interference between the short compressed pulse and the spectrally-narrow pedestal.

This technique is demonstrated with $10-$ and $20-\mathrm{GHz}$ mode spacing, but would work equally well at repetition rates from 5 to $30 \mathrm{GHz}$ or more (peak power and pulse width should be kept similar, upper frequency limited by stimulated Brillouin scattering). The wavelength tunability is only restricted by the efficiency of the DDF compression, but will certainly operate over most of the C-band. The demonstrated system can achieve wall plug power much less than $10 \mathrm{~W}$ including the $22 \mathrm{dBm}$ EDFA. The scalability to $\sim 1000$ optical frequency comb is possible with increased EDFA saturation power beyond $30 \mathrm{dBm}$. Reduction in power and size of the future off-chip OFCG is possible through monolithic 


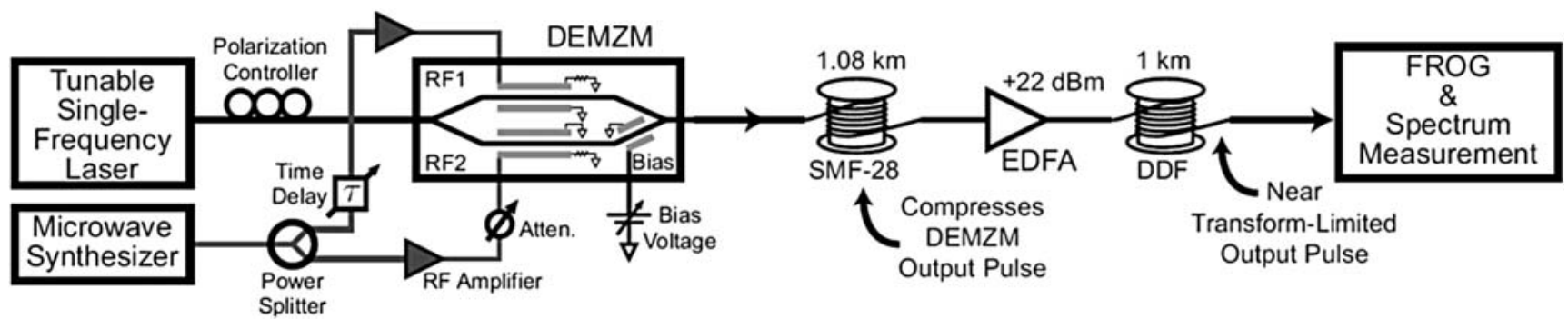

Fig. 9 Experimental arrangement for widely tunable optical frequency comb generation using soliton-like compression in DDF. DEMZM: dual-electrode Mach-Zehnder modulator

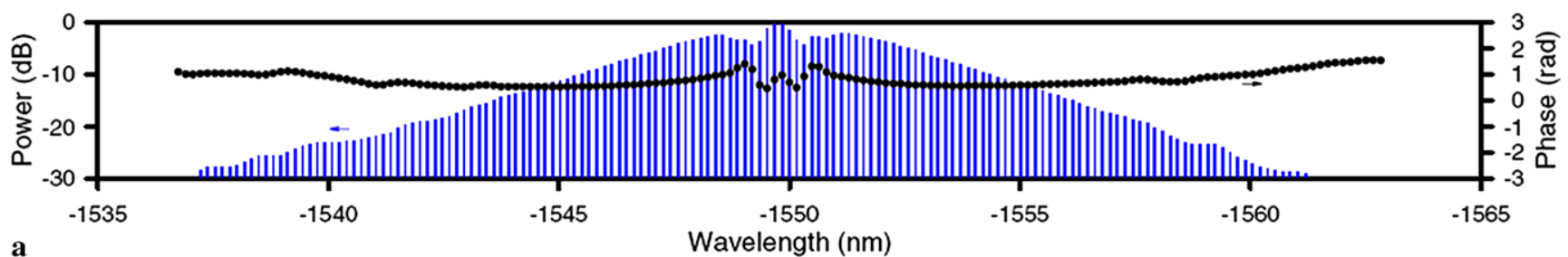

a

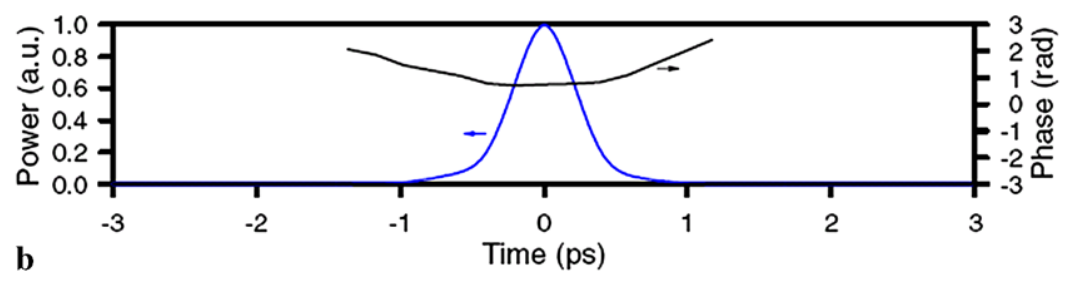

Fig. 10 Output from DDF retrieved by FROG showing nearly flat spectral phase. (a) Retrieved spectral mode intensity (solid lines) and phase (filled circles). (b) Retrieved temporal intensity (blue) and phase (black)
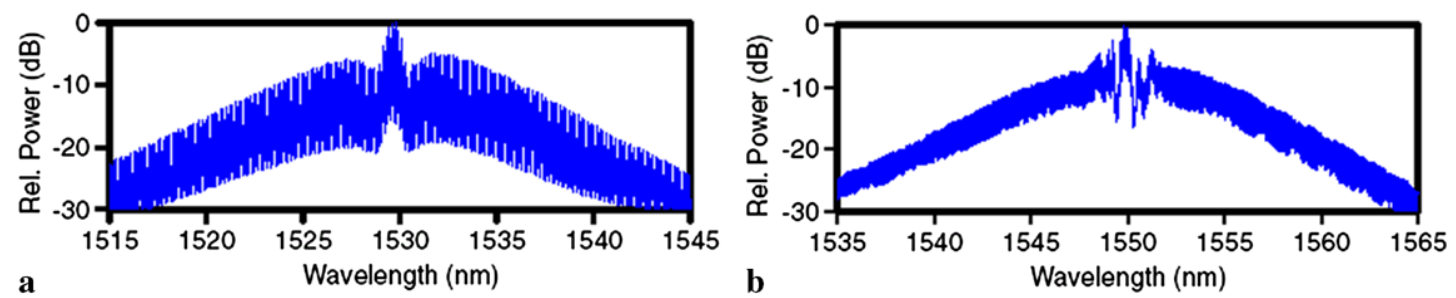

Fig. 11 Spectrum of the DDF output demonstrating the OFCG tunability. (a) Tunable laser output centered at $1530 \mathrm{~nm}(20 \mathrm{GHz}$ mode spacing). (b) $10 \mathrm{GHz}$ mode spacing centered at $1550 \mathrm{~nm}$. OSA resolution is $0.05 \mathrm{~nm}$ or $6.25 \mathrm{GHz}$

integration of the laser, modulator, and nonlinear optical element (e.g., semiconductor optical amplifier) all on an identical semiconductor platform (e.g., InP).

\section{Computer system applications}

Due to their low latency and flexibility, crossbar based onchip interconnection networks are useful in many computing systems such as multicore processors, complex systems-onchip and programmable logic devices. With the ability to transmit multiple wavelengths over the same waveguide and the use of nanoscale microring resonators, large nanopho- tonic crossbars can be realized that offer low power consumption and high performance [45]. However, silicon microring resonators are very sensitive to the ambient temperature change, as silicon has a relatively large thermo-optic coefficient. The non-uniform temperature distribution (so called hotspots) in a high-performance multicore processor presents a serious impediment to the realization of nanophotonic interconnect networks based on the silicon microring resonator.

We use Hotspot v4.1 [46] from University of Virginia to generate the thermal profile of a 64-core processor array that is based on the Corona architecture [11]. We modeled a generic 3D architecture shown in Fig. 1. The bottom layer 
Fig. 12 Temperature contour map for a 64-core processor. The temperature is shown in degrees Kelvin

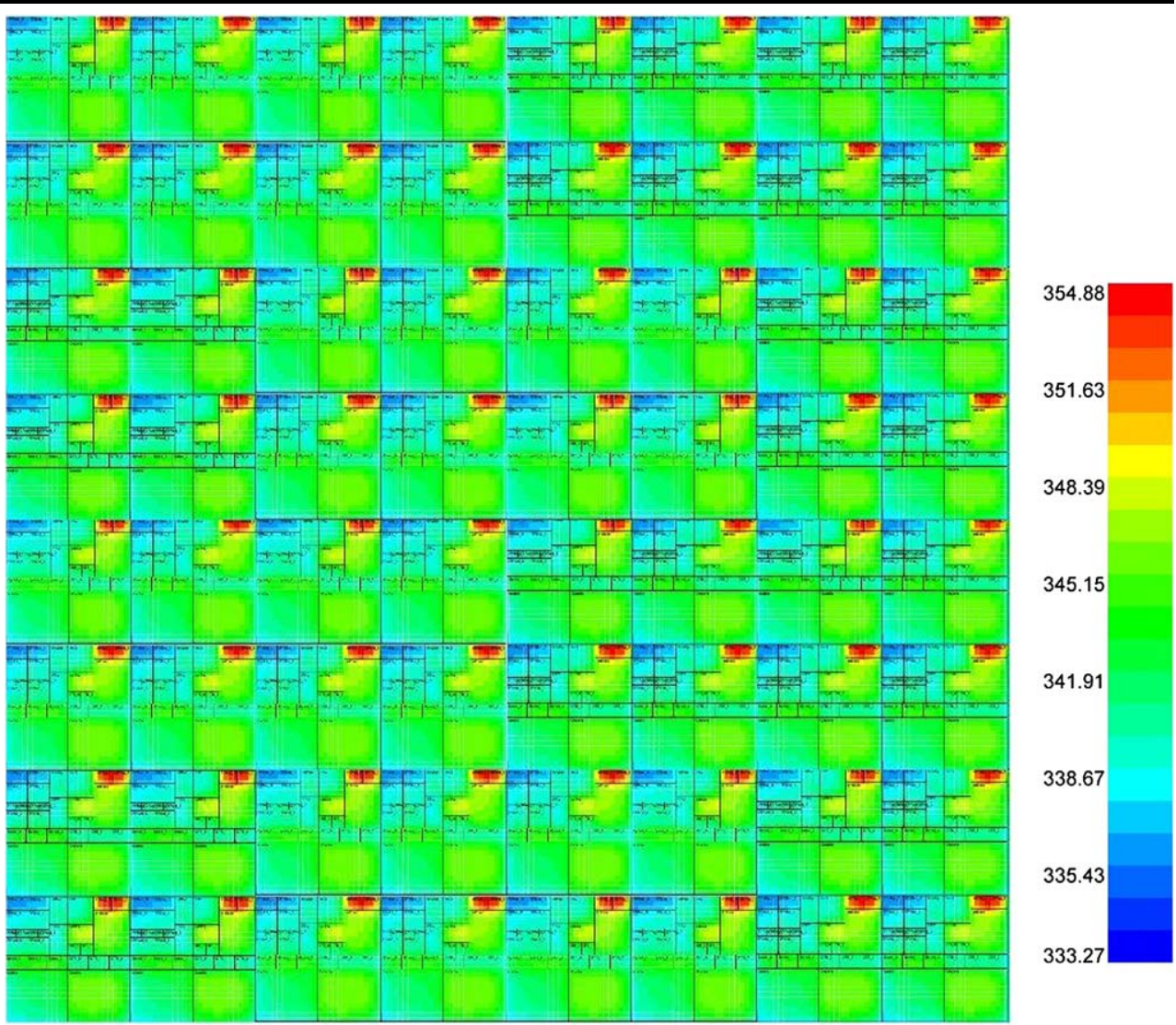

consists of 64 cores modeled after Alpha EV6, including the L1 caches. The middle layer consists of a large shared L2 cache. The optical interconnect is assumed to reside on the top layer. The processors were at $3 \mathrm{GHz}$ and we assumed a $100 \mathrm{~nm}$ technology node. Figure 12 shows the temperature contour map of the 64 -core processor. The temperature changes from 60 to $82^{\circ} \mathrm{C}$ across the whole chip. The technique proposed in this paper to build athermal microring resonators can be used to achieve stable operation across the operating temperature range without requiring individual temperature sensors and thermal controllers on each silicon microresonator.

\section{Summary}

This paper demonstrates that temperature sensitivity of slotted microring resonators can be reduced by using PMMA upper cladding, as PMMA has negative thermo-optic (TO) coefficient which can be used to compensate for the positive coefficient of silicon material. Simulations showed that temperature insensitive waveguides can be designed with slot infiltrated with PMMA or even air. Preliminary experimental results demonstrated that the temperature dependence can be reduced from $91 \mathrm{pm} /{ }^{\circ} \mathrm{C}$ for a regular microring resonator to $67 \mathrm{pm} /{ }^{\circ} \mathrm{C}$ for the slotted microring resonator with- out any upper-cladding and further reduced to $52 \mathrm{pm} /{ }^{\circ} \mathrm{C}$ for PMMA cladding. The external optical source utilized a 3.5-THz-wide optical frequency comb generator with wavelength and comb spacing tunable. Nearly flat spectral phase of the output spectra was achieved by DDF compression of a non-resonant OFCG and results in 175 optical modes with $20 \mathrm{GHz}$ spacing, and 300 optical modes with $10 \mathrm{GHz}$ spacing, tunable over most of the C-band. The flat spectral phase, simplicity and tunability make this source very attractive for optically-interconnected computing system applications of the future.

Open Access This article is distributed under the terms of the Creative Commons Attribution Noncommercial License which permits any noncommercial use, distribution, and reproduction in any medium, provided the original author(s) and source are credited.

\section{References}

1. G. Bell, J. Gray, A. Szalay, Computer 39, 110 (2006)

2. D.A.B. Miller, A. Bhatnagar, S. Palermo, A. Emami-Neyestanak, M.A. Horowitz, in IEEE International Solid-State Circuits Conference (ISSCC) (2005), p. 86

3. D.A.B. Miller, Proc. IEEE 88, 728 (2000)

4. R.G. Beausoleil, in IEEE LEOS Annual Meeting, Paper \#WM3, Orlando Florida (2007)

5. J. Shah, in DARPATECH, Anaheim, California (2007) 
6. D.A.B. Miller, Proc. IEEE 88, 728 (2000)

7. F. Benner, M. Ignatowski, J.A. Kash, D.M. Kuchta, M.B. Ritter, IBM J. Res. Dev. (2005)

8. J. Bautista, in Interconnect Focus Center, Quarterly Workshop, Stanford University (2006)

9. A. Hadke, T. Benavides, S.J.B. Yoo, R. Amirtharajah, V. Akella, in Proceedings of 16th IEEE Symposium on High Performance Interconnects (HOTI 2008), Palo Alto, CA (2008)

10. A. Hadke, T. Benavides, M. Farrens, R. Amirtharajah, V. Akella, in Proceedings of 26th IEEE International Conference on Computer Design, Squaw Creek, Lake Tahoe, CA, Oct. (2008)

11. D. Vantrease et al., in Proceedings of 35th International Symposium on Computer Architecture (ISCA'08) (2008), p. 153

12. C. Batten et al., in Proceedings of 16th IEEE Symposium on High Performance Interconnects (HOTI '08) (2008), p. 21

13. A. Shacham, K. Bergman, L.P. Carloni, IEEE Trans. Comput. 57, 1246 (2008)

14. R.G. Beausoleil, P.J. Kuekes, G.S. Snider, S.Y. Wang, R.S. Williams, Proc. IEEE 96, 230 (2008)

15. A. Shacham, K. Bergman, L.P. Carloni, in Proceedings of the First IEEE International Symposium on Networks-on-Chips, Institute of Electrical and Electronics Engineers, New York (2007), p. 53

16. M.L. Brongersma, Nat. Photonics 2, 270 (2008)

17. H. Fischer, O.J.F. Martin, Opt. Express 16, 9144 (2008)

18. T. Barwicz et al., J. Opt. Netw. 6, 63 (2007)

19. C. Gunn, IEEE MICRO 58 (2006)

20. M.R. Wang, H.Y. Ng, D. Li, X. Wang, J. Martinez, R.R. Panepucci, K. Pathak, in Proceedings of the SPIE (2007), p. 66450I

21. S. Janz et al., IEEE J. Sel. Top. Quantum Electron. 12, 1402 (2006)

22. V.R. Almeida, Q. Xu, C.A. Barrios, M. Lipson, Opt. Letters 29, 1209 (2004)

23. P.A. Anderson, B.S. Schmidt, M. Lipson, Opt. Express 14, 9197 (2006)

24. T. Baehr-Jones, M. Hochberg, G. Wang, R. Lawson, Y. Liao, P. Sullivan, L. Dalton, A. Jen, A. Scherer, Opt. Express 13, 5216 (2005)

25. F. Dell'Olio, V.M. Passaro, Opt. Express 15, 4977 (2007)

26. A. Di Falco, L. O'Faolain, T.F. Krauss, Appl. Phys. Lett. 92, 083501 (2008)
27. N.N. Feng, R. Sun, L.C. Kimerling, J. Michel, Opt. Lett. 32, 1250 (2007)

28. N.N. Feng, R. Sun, J. Michel, L.C. Kimerling, Opt. Lett. 32, 2131 (2007)

29. M. Galli et al., Appl. Phys. Lett. 89, 241114 (2006)

30. M. Hochberg, T. Baehr-Jones, G. Wang, J. Huang, P. Sullivan, L. Dalton, A. Scherer, Opt. Express 15, 8401 (2007)

31. J.M. Lee, D.J. Kim, G.H. Kim, O.K. Kwon, K.J. Kim, G. Kim, Opt. Express 16, 1645 (2008)

32. C. Ma, Q. Zhang, E. Van Keuren, Opt. Express 16, 14330 (2008)

33. J.T. Robinson, L. Chen, M. Lipson, Opt. Express 16, 4296 (2008)

34. P. Sanchis, J. Blasco, A. Martinez, J. Marti, J. Lightw. Technol. 25, 1298 (2007)

35. G. Wang, T. Baehr-Jones, M. Hochberg, A. Scherer, Appl. Phys. Lett. 91, 143109 (2007)

36. J. Xiao, X. Liu, X. Sun, Opt. Express 15, 8300 (2007)

37. Q. Xu, V.R. Almeida, R.R. Panepucci, M. Lipson, Opt. Letters 29, 1626 (2004)

38. S.H. Yang, M.L. Cooper, P.R. Bandaru, S. Mookherjea, Opt. Express 16, 8306 (2008)

39. K. Kashiwagi, K. Okamoto, S.J.B. Yoo, in European Conference on Integrated Optics (2008), Paper FrD4

40. B.R. Koch, A.W. Fang, O. Cohen, J.E. Bowers, Opt. Express 15, 11225 (2007)

41. M. Kourogi, K. Nakagawa, M. Ohtsu, IEEE J. Quantum Electron. 29, 2693 (1993)

42. T. Sakamoto, T. Kawanishi, M. Izutsu, in Proceedings of the Conference on Lasers and Electro-Optics (CLEO 2006) (2006), p. CMAA5

43. N.K. Fontaine, R.P. Scott, W. Cong, B.H. Kolner, J.P. Heritage, S.J.B. Yoo, in Quantum Electronics and Laser Science Conference, 2005 (QELS '05) (2005), p. 1328

44. N.K. Fontaine, R.P. Scott, J. Cao, K. Okamoto, J.P. Heritage, B.H. Kolner, S.J.B. Yoo, in Proceedings of the Conference on Lasers and Electro-Optics (CLEO'06) (2006), p. We4.6.7

45. A. Shacham, K. Bergman, L.P. Carloni, in Design Automation Conference, 2007. DAC '07. 44th ACM/IEEE (2007), p. 132

46. http://lava.cs.virginia.edu/HotSpot/ 